\title{
Pairwise Strongly Lindelöf, Pairwise Nearly, Almost and Weakly Lindelöf Bitopological Spaces
}

\author{
EMAN ALMUHUR ${ }^{1}$, MANAL AL-LABADI ${ }^{2}$ \\ ${ }^{1}$ Department of Basic Science and Humanities, Faculty of Arts and Science \\ Applied Science Private University \\ P.O. Box 166, Amman 11931, JORDAN \\ ${ }^{2}$ Department of Mathematics, Faculty of Arts and Sciences \\ University of Petra \\ P.O. Box 660, Amman 961343, JORDAN
}

\begin{abstract}
The main purposes of this article is to introduce new generalizations of the notion of pairwise Lindelöf spaces in bitopological spaces where new notions: pairwise strongly Lindelöf, pairwise nearly, pairwise almost and pairwise weakly strongly Lindelöf bitopological spaces depend on the new notion pairwise preopen countable covers. These covers where we focused on their importance in topology consist of countable subfamilies whose closures cover the bitopological spaces and we clarified how pairwise preopen countable covers effect on pairwise strongly Lindelöf spaces. The new concepts of pairwise strongly Lindelöf, pairwise nearly, pairwise almost and pairwise weakly strongly Lindelöf bitopological spaces are introduced and many definitions, propositions, characterizations and remarks concerning those notions are initiated, discussed and explored. Furthermore, the relationships between those bitopological spaces are examined and investigated. We illustrated the implications hold by these new bitopological spaces. We put some queries and claims, then we struggle to provide their proofs.
\end{abstract}

Key-Words: - Pairwise Strongly Lindelöf, Pairwise Almost Strongly Lindelöf.

Received: December 12, 2020. Revised: March 15, 2021. Accepted: March 31, 2021. Published: April 26, 2021.

\section{Introduction}

The study of bitopological spaces was initiated by J.C.Kelly [1] in 1963 in an article in London mathematical society titled "Bitopological spaces" and thereafter a lot of articles were proposed to generalize topological concepts to bitopological ones. Typically, If $X$ is a non-empty set endowed with two different topologies $\tau_{1}$ and $\tau_{2}$ on it, then the triple $\left(X, \tau_{1}, \tau_{2}\right)$ or simply $X$ is said to be a bitopological space. Mashhour [2] initiated a topological space named strongly compact (resp. Lindelöf) in which every preopen cover of a space has a finite (resp. countable) subcover. After there, many articles discussing such spaces introduced by Ganster [3], Omari, Noiri and Noorani [4]. In addition, Hdeib and Sarsak [5] proposed new results concerning those notions. Examining the relationship between strongly compact spaces and strongly lindelöf spaces reveals a lot of class of such spaces. In a topological space $(X, \tau)$, a subset $U$ is called preopen [6] if it is contained in the interior of its closure, hence every open subset of a topological space is preopen. Moreover, every dense subset of a topological space is preopen.

Ganster [7] showed that a subset $U$ of $X$ is preopen if it is the intersection of an open and dense subset. Few years later, Ganster [3] introduced the notion of dLindelöf spaces. In this space, each cover of a space $X$ by dense subsets has a countable subcover. He pointed out that a topological space $X$ is d- Lindelöf if and only if $X-I_{X}$ is countable where $I_{X}$ is the set of all isolated points of a space $X$, or equivalently, $\bar{U}-U$ is countable $\forall U \subseteq X$. Typically, every strongly Lindelöf space is Lindelöf. Hdeib and Sarsak [8] called a subset $U$ of a topological space $(X, \tau)$ strongly Lindelöf relative to $X$ if each preopen cover of $U$ admits a countable subcover of $U$. Some relevant studies can be found in [20] and [21]. 


\section{Preliminaries}

Salleh in his paper [9] called a subset $U$ of $X$ in the bitopological space $\left(X, \tau_{1}, \tau_{2}\right)$ an $(i, j)$-preopen (resp. $(i, j)$-preclosed) if

$U \subseteq i-\operatorname{int}(j-c l(U)) \quad($ resp. $\quad i-c l(j-$ $\operatorname{int}(U)) \forall i, j=1,2 \quad i \neq j$.

If $U$ is $(1,2)$-preopen and $(2,1)$-preopen, then $U$ is pairwise preopen. The complement of a pairwise preopen subset is pairwise preclosed. Furthermore, Salleh [16] called a subset $O$ of a bitopological space $\left(X, \tau_{1}, \tau_{2}\right)$ an $(i, j)$-regular open (resp. $(i, j)$-regular closed) if

$O=i-\operatorname{int}(j-\operatorname{cl}(O))($ resp. $\quad O=i-\operatorname{cl}(j-$ $\operatorname{int}(0))$. If $O$ is $(1,2)$-regular open and $(2,1)$-regular open, then $O$ is pairwise regular open. The complement of a pairwise regular open is pairwise regular closed. A subset $A$ of a bitopological space $\left(X, \tau_{1}, \tau_{2}\right)$ is said to be $(i, j)$-semi-open (resp. $(i, j)$-semi-closed) if

$A \subseteq i-\operatorname{cl}(j-\operatorname{int}(A))$

(resp. $A \subseteq i-\operatorname{int}(j-$ $c l(A))$ see [11]. If $A$ is $(1,2)-$ semi-open and $(2,1)$-semi-open, then $A$ is a pairwise semi-open. The complement of a pairwise semi-open subset is pairwise semi-closed.

\section{Pairwise Strongly Lindelöf}

A bitopological space $\left(X, \tau_{1}, \tau_{2}\right)$ is said to be $(i, j)$-strongly compact if for every $(i, j)$-preopen cover $\left\{u_{\alpha}: \alpha \in \Lambda\right\}$ of $X$, there exists a finite subset $\left\{\alpha_{1}, \alpha_{2}, \ldots, \alpha_{k}\right\}_{k \in \mathbb{N}}$ of $\Lambda$ such that $X=$ $\bigcup_{n=1}^{k} u_{\alpha_{n}} \forall i, j=1,2 \quad i \neq j$. A bitopological space is called pairwise strongly compact if it is $(1,2)$-strongly compact and $(-2,1)$-strongly compact.

A bitopological space $\left(X, \tau_{1}, \tau_{2}\right)$ is said to be $(i, j)$-strongly Lindelöf if for every $(i, j)$-preopen cover $\left\{u_{\alpha}: \alpha \in \Lambda\right\}$ of $X$, there exists a countable subset $\left\{\alpha_{n}: n \in \mathbb{N}\right\}$ of $\Lambda$ such that $X=$ $\bigcup_{n \in \mathbb{N}}\left(u_{\alpha_{n}}\right) \quad \forall i, j=1,2 \quad i \neq j$. A topological space is called pairwise strongly Lindelöf if it is $(1,2)$-strongly Lindelöf and $(2,1)$-strongly Lindelöf.

If $U$ is a subset of a bitopological space $\left(X, \tau_{1}, \tau_{2}\right)$, then $U$ is said to be $(i, j)$-strongly Lindelöf relative to $X$ if every $(i, j)$-preopen cover of $U$ admits a countable subcover of it $\forall i, j=1,2 \quad i \neq j$. A subset $U$ is pairwise strongly Lindelöf relative to $X$ if it is both $(1,2)$-strongly Lindelöf relative to $X$ and $(2,1)$-strongly Lindelöf relative to $X$.

Remark3.1: If a subset $U$ of a bitopological space $\left(X, \tau_{1}, \tau_{2}\right)$ is pairwise strongly Lindelöf relative to $X$, then $U$ is pairwise strongly Lindelöf. Bourbaki [13] called a topological space $(X, \tau)$ submaximal if each of its dense subsets is open. Reilly and Vamanamurthy [10] proved that $X$ is submaximal if and only if each preopen subset of $X$ is open. Ganster [4] showed that each submaximal Lindelöf space is strongly Lindelöf. A bitopological space $\left(X, \tau_{1}, \tau_{2}\right)$ is said to be $(i, j)$-submaximal if each $i$-dense subset of $X$ is $j$-open $\forall i, j=1,2 \quad i \neq j$. If $X$ is $(1,2)$-submaximal and $(2,1)$-submaximal, then $X$ is pairwise submaximal.

A bitopological space $\left(X, \tau_{1}, \tau_{2}\right)$ is said to be a pairwise maximal strongly Lindelöf if it is pairwise strongly Lindelöf and there exists no strictly finer pairwise strongly Lindelöf bitopological space.

Proposition3.2: A bitopological space is pairwise maximal strongly Lindelöf if and only if it is pairwise L-closed.

Proposition3.3: A countable discrete bitopological space is a pairwise maximal strongly Lindelöf.

Recall that a bitopological space $\left(X, \tau_{1}, \tau_{2}\right)$ is $\mathrm{p}$ Housdorff [16] if for each distinct points $x$ and $y$ in $X$, there exist two disjoint subsets $u \in\left(X, \tau_{i}\right)$ and $v \in$ $\left(X, \tau_{j}\right)$ such that $x \in u$ and $y \in v \forall i, j=1,2 \quad i \neq j$. Moreover, a bitopological space $\left(X, \tau_{1}, \tau_{2}\right)$ is a pairwise $\mathrm{P}$-space if a countable intersection of $\tau_{i}$-open subsets of $X$ is $\tau_{j}$-open subset $\forall i, j=$ $1,2 i \neq j$.

Proposition3.4: If a bitopological space $\left(X, \tau_{1}, \tau_{2}\right)$ is p-Housdorff pairwise strongly Lindelöf, then $X$ is a pairwise maximal strongly Lindelöf if and only if it is a pairwise $\mathrm{P}$-space.

Recall that a bitopological space $\left(X, \tau_{1}, \tau_{2}\right)$ is $\mathrm{p}$ normal if for a given $\tau_{1}$-closed subset $F_{1}$ and a $\tau_{2}$-closed subset $F_{2}$ of $X$ such that $F_{1} \cap F_{2}=\emptyset$, there exists a $\tau_{1}$-open subset $u$ and a $\tau_{2}$-open subset $v$ such that $F_{1} \subseteq u$ and $F_{2} \subseteq v$ and $u \cap v=\emptyset$. 
Proposition3.5: If a bitopological space $\left(X, \tau_{1}, \tau_{2}\right)$ is p-Hausdorff pairwise maximal strongly Lindelöf , then $X$ is p-normal.

Proposition3.6: Every pairwise maximal strongly Lindelöf is a pairwise submaximal.

Proposition3.7: If a bitopological space $\left(X, \tau_{1}, \tau_{2}\right)$ is p-Housdorff pairwise strongly Lindelöf, then it is a pairwise strongly Lindelöf if and only if it is a Pspace.

Proposition3.8: A bitopological space $\left(X, \tau_{1}, \tau_{2}\right)$ is pairwise submaximal if and only if $X$ is pairwise open and pairwise preopen.

Proposition3.9: The set of all rational numbers is pairwise Lindelöf but not pairwise submaximal.

Proposition3.10: Every pairwise strongly Lindelöf is a pairwise Lindelöf.

Proof: Suppose that a bitopological space $\left(X, \tau_{1}, \tau_{2}\right)$ is pairwise strongly Lindelöf, and let $\left\{u_{\alpha}: \alpha \in \Lambda\right\}$ be a cover consisting of pairwise preopen subsets, then $\left\{u_{\alpha}: \alpha \in \Lambda\right\}$ is a cover consisting of pairwise open subsets since each preopen subset is open. Hence, there exists a countable subset $\left\{\alpha_{1}, \alpha_{2}, \ldots\right\}$ such that $X=\bigcup_{k=1}^{\infty} u_{\alpha_{k}}$.That is, $X$ is pairwise Lindelöf.

\section{Pairwise Nearly Strongly Lindelöf Spaces}

Proposition4.1: In a bitopological space $\left(X, \tau_{1}, \tau_{2}\right)$, $X$ is pairwise nearly strongly Lindelöf if and only if for each family $\left\{F_{\alpha}: \alpha \in \Lambda\right\}$ of pairwise preclosed subsets of $X$ with $\bigcap_{\alpha \in \Lambda} F_{\alpha} \neq \varnothing$, there exists a countable subset $\left\{\alpha_{1}, \alpha_{2}, \ldots\right\}$ such that $\bigcap_{k \in \mathbb{N}} \operatorname{precl}_{i}\left(\right.$ preint $\left._{j} F_{\alpha_{k}}\right) \neq \emptyset$.

Proof: Let $X$ be a pairwise nearly strongly Lindelöf and that a family $\left\{F_{\alpha}: \alpha \in \Lambda\right\}$ of pairwise preclosed subsets of $X$ has a non-empty intersection, then

$X=X-\bigcap_{\alpha \in \Lambda} F_{\alpha}=\cup_{\alpha \in \Lambda}\left(X-F_{\alpha}\right)$. So, the family $\left\{F_{\alpha}: \alpha \in \Lambda\right\}$ is a pairwise preopen cover of $X$. The bitopological space being pairwise nearly strongly Lindelöf, thus there exists a countable subset $\left\{\alpha_{1}, \alpha_{2}, \ldots\right\}$ such that

$$
X=\bigcup_{k \in \mathbb{N}} \operatorname{preint}_{i}\left(\operatorname{precl}_{j}\left(X-F_{\alpha_{k}}\right)\right)=
$$

$\bigcup_{k \in \mathbb{N}} \operatorname{preint}_{i}\left(X-\operatorname{precl}_{j}\left(F_{\alpha_{k}}\right)\right)=\bigcup_{k \in \mathbb{N}}(X-$

$\left.\operatorname{precl}_{i}\left(\operatorname{preint}_{j}\left(F_{\alpha_{k}}\right)\right)\right)=X-$

$\bigcap_{k \in \mathbb{N}} \operatorname{precl}_{i}\left(\operatorname{preint}_{j}\left(F_{\alpha_{k}}\right)\right)$.

Thus, $\bigcap_{k \in \mathbb{N}} \operatorname{precl}_{i}\left(\operatorname{preint}_{j}\left(F_{\alpha_{k}}\right)\right) \neq \varnothing$

For the converse, suppose that for each family $\left\{F_{\alpha}: \alpha \in \Lambda\right\}$ of pairwise preclosed subsets of $X$ with $\bigcap_{\alpha \in \Lambda} F_{\alpha} \neq \varnothing$, there exists a countable subset $\left\{\alpha_{1}, \alpha_{2}, \ldots\right\}$ such that $\bigcap_{k \in \mathbb{N}}$ precl $_{i}\left(\right.$ preint $\left._{j} F_{\alpha_{k}}\right) \neq \varnothing$, let $\left\{u_{\alpha}: \alpha \in \Lambda\right\}$ be a pairwise preopen cover of $X$, then $\varnothing \neq X-\cup_{\alpha \in \Lambda} u_{\alpha}$, so $\left\{X-u_{\alpha}: \alpha \in \Lambda\right\}$ is a family of pairwise preclosed subsets that has a nonempty intersection, by the assumption, there exists a countable subset such that $\left\{X-u_{\alpha_{k}}: k \in \mathbb{N}\right\}$ such that $\quad \varnothing \neq \bigcap_{k \in \mathbb{N}} \operatorname{precl}_{i}\left(\operatorname{preint}_{j}\left(X-u_{\alpha_{k}}\right)\right)=$ $\bigcap_{k \in \mathbb{N}} \operatorname{precl}_{i}\left(X-\operatorname{preint}_{j}\left(u_{\alpha_{k}}\right)\right)=X$ pre-

$\cup_{k \in \mathbb{N}} \operatorname{preint}_{i}\left(\operatorname{precl}_{j}\left(u_{\alpha_{k}}\right)\right)$, so

$X=\cup_{k \in \mathbb{N}} \operatorname{preint}_{i}\left(\operatorname{precl}_{j}\left(u_{\alpha_{k}}\right)\right)$, that is, $X$ is a pairwise nearly strongly Lindelöf.

Proposition4.2: If a bitopological space $\left(X, \tau_{1}, \tau_{2}\right)$ is a p-regular and every point has a pairwise nearly strongly Lindelöf neighbourhood, then $X$ is pairwise nearly strongly Lindelöf.

Proof: Let $F$ be a pairwise nearly strongly Lindelöf subset of $X$ and suppose that $x \in X \quad \ni$

$$
x \in \operatorname{preint}_{i}\left(\operatorname{precl}_{j}(F)\right)-F \forall i, j=1,2 i \neq j,
$$

then $\exists$ a pairwise preopen subset $u$ containing $x$ and a preopen set $v$ containing $F$.

Now, $u$ is a pairwise nearly strongly Lindelöf neighbourhood of $x$. But $X$ is p-regular, so $\exists$ a preopen subset $w \ni$

$x \in w \subseteq \operatorname{preint}_{i}\left(\operatorname{precl}_{j}(w)\right) \subseteq u \quad$ and $\operatorname{preint}_{i}\left(\operatorname{precl}_{j}(F)\right) \cap \operatorname{preint}_{i}\left(\operatorname{precl}_{j}(w)\right)$ is a pairwise nearly strongly Lindelöf subset of $u$. Therefore, $x \in F$ and $F$ is pairwise closed.

For every pairwise preopen cover $\left\{u_{\beta}: \beta \in \Lambda\right\}$ of $F$, there exists a pairwise countable subset $\left\{\beta_{1}, \beta_{2}, \ldots\right\}$ such that $F \subseteq \bigcup_{k \in \mathbb{N}} \operatorname{preint}_{i}\left(\operatorname{precl}_{j}\left(u_{\beta_{k}}\right)\right)$. Thus, $X$ is a pairwise nearly strongly Lindelöf.

Proposition4.3: In a bitopological space $\left(X, \tau_{1}, \tau_{2}\right)$, the following statements are equivalent: 
1. $X$ is pairwise nearly strongly Lindelöf.

2. For every family of p-regular closed subsets $\left\{F_{\alpha}: \alpha \in \Delta\right\}$ of $X \ni \cap F_{\alpha}=\varnothing$,

there is a countable subset $\left\{\alpha_{n}: n \in \mathbb{N}\right\}$ of $\Delta \ni$ $\bigcap_{n \in \mathbb{N}} F_{\alpha_{n}}=\emptyset$.

Proposition4.4: If a bitopological space $\left(X, \tau_{1}, \tau_{2}\right)$ is pairwise nearly strongly Lindelöf, then for each pairwise regularly preclosed subsets $A$ and $B$ of $X, \exists$ two disjoint preopen subsets $U$ and $V \ni A \subseteq U$ and $B \subseteq V$.

Proof: Let $X$ be a pairwise nearly strongly Lindelöf p-regular space, then for every $x \in A, \exists$ a pairwise preopen $x$-neighborhood $U_{x} \ni B \subseteq X-c l_{i}\left(U_{x}\right)$ $\forall i=1,2$.

If $\widetilde{U}=\left\{U_{x}: x \in A\right\}$ is a pairwise preopen-cover of $X$, then $\exists$ a countable set of points of $X$ say $\left\{x_{1}, x_{2}, \ldots\right\} \ni \quad X=\left(\cup_{k=1}^{\infty} \operatorname{int}_{i}\left(\operatorname{cl}_{j}\left(U_{x_{k}}\right)\right)\right) \cup(X-$ $A$ ) because $X$ is a pairwise nearly strongly Lindelöf space $\forall i, j=1,2 \quad i \neq j$.

Similarly, for every $y \in B, \exists$ a pairwise preopen $y$-neighborhood $G_{y} \ni B \subseteq X-c l_{i}\left(G_{y}\right) \forall i=1,2$. If $\widetilde{G}=\left\{G_{y}: y \in B\right\}$ is a pairwise preopen-cover of $X$, then $\exists$ a countable set of points of $X$ say $\left\{y_{1}, y_{2}, \ldots\right\} \ni$ $X=\left(\cup_{k=1}^{\infty} \operatorname{int}_{i}\left(c l_{j}\left(G_{y_{k}}\right)\right)\right) \cup(X-B)$ because $X$ is a pairwise nearly strongly Lindelöf. Now, let $L_{k}=$ $O_{x_{k}}-\cup_{k=1}^{\infty} c l_{i}\left(G_{y_{k}}\right)$ and $M_{k}=G_{y_{k}}-\cup_{k=1}^{\infty} c l_{i}\left(O_{x_{k}}\right)$, then $O=\bigcup_{k=1}^{\infty} L_{k}$ and $G=\bigcup_{k=1}^{\infty} M_{k}$ are two disjoint pairwise preopen subsets of $X$.

Proposition4.5: In a bitopological space $\left(X, \tau_{1}, \tau_{2}\right)$, the sum $\oplus_{\alpha \in \Lambda} X_{\alpha}$ where $X_{\alpha} \neq \varnothing$ for some $\alpha \in \Lambda$ has pairwise nearly strongly Lindelöf property if and only if all bitopological spaces $X_{\alpha}$ have the pairwise nearly strongly Lindelöf property and the set $\Lambda$ is countable.

Proof: Suppose that the sum $\oplus_{\alpha \in \Lambda} X_{\alpha}$ where $X_{\alpha} \neq \varnothing$ for some $\alpha \in \Lambda$ has pairwise nearly strongly Lindelöf property, then all bitopological spaces $X_{\alpha}$ are pairwise nearly strongly Lindelöf the set $\Lambda$ is countable.

On the other hand, suppose that all bitopological spaces $X_{\alpha}$ have the pairwise nearly strongly Lindelöf property and the set $\Lambda$ is countable and
$\left\{\left(X, \tau_{1}{ }^{k}, \tau_{2}{ }^{k}\right): k \in \mathbb{N}\right\}$ is a family of pairwise nearly strongly Lindelöf, then $X=X_{1} \oplus X_{2} \oplus \ldots$ is pairwise nearly strongly Lindelöf.

Example4.6: Let $X=\mathbb{N} \times[0,1]$ and the topology on $X$ is a subspace of the plane. Now, every $\{a\} \times[0,1]$ is open and closed for every $a \in \mathbb{N}$ in $X$. A space $X$ can be expressed as a sum of many copies of $[0,1]$.

Proposition4.7: If a bitopological space $\left(X, \tau_{1}, \tau_{2}\right)$ is pairwise nearly strongly Lindelöf and a subset $A$ of $X$ is pairwise locally countable subset of $X$, then $A$ is discrete.

5.Pairwise Almost Strongly Lindelöf Spaces A bitopological space $\left(X, \tau_{1}, \tau_{2}\right)$ is called an $\tau_{i}$-almost strongly Lindelöf if every pairwise preopen cover $\left\{u_{\alpha}: \alpha \in \Lambda\right\}$ of $X$ admits a countable subset $\left\{\alpha_{1}, \alpha_{2}, \ldots\right\}$ such that $X=$ $\bigcup_{k \in \mathbb{N}} \operatorname{precl}_{i}\left(u_{\alpha_{k}}\right) \forall i=1,2$. Now if $X$ is $\tau_{1}$-almost strongly Lindelöf and $\tau_{2}$-almost strongly Lindelöf, then $X$ is a pairwise almost Lindelöf.

Proposition5.1: A bitopological space $\left(X, \tau_{1}, \tau_{2}\right)$ is a pairwise nearly strongly, pairwise almost strongly Lindelöf if and only if every family of a pairwise preclosed subsets $\tilde{F}=\left\{F_{\alpha}: \alpha \in \Delta\right\}$ of $X$ such that $\bigcap_{\alpha \in \Delta} F_{\alpha}=\emptyset$ admits a countable subfamily such that $\bigcap_{k=1}^{\infty} F_{\alpha_{k}}=\emptyset$. Proof: Suppose that $\tilde{F}=\left\{F_{\alpha}: \alpha \in \Delta\right\}$ is a family of pairwise preclosed subsets of $X$ such that $\bigcap_{\alpha \in \Delta} F_{\alpha}=$ $\emptyset$, then $\left\{X-F_{\alpha}: \alpha \in \Delta\right\}$ is a cover of $X$ consisting of pairwise preopen subsets.

Now, $X$ is a pairwise nearly strongly and a pairwise almost strongly Lindelöf space, so there exists a countable subfamily $\left\{\alpha_{1}, \alpha_{2}, \ldots\right\}$ such that $X=$ $\cup_{k=1}^{\infty} \operatorname{precl}_{i}\left(\left(X-F_{\alpha_{k}}\right)\right)$, that is $\bigcap_{k=1}^{\infty} \operatorname{preint}_{i}\left(F_{\alpha_{k}}\right)=\emptyset$.

On the other hand, if $\widetilde{U}=\left\{u_{\alpha}: \alpha \in \Delta\right\}$ is a pairwise preopen cover of $X$, then $\left\{X-u_{\alpha}: \alpha \in \Delta\right\}$ is a family of pairwise preclosed subsets of $X$ such that $\bigcap_{\alpha \in \Delta}\left(X-u_{\alpha}\right)=\varnothing$. Using the assumption, there exists a countable subfamily $\left\{\alpha_{1}, \alpha_{2}, \ldots\right\}$ such that $\bigcap_{k=1}^{\infty}\left(X-u_{\alpha_{k}}\right)=$

$$
\emptyset \text {, i.e } X=\cup_{k=1}^{\infty} \operatorname{cl}\left(u_{\alpha_{k}}\right) \text {. }
$$

Propositio5.2: A bitopological space $\left(X, \tau_{1}, \tau_{2}\right)$ is a pairwise almost strongly Lindelöf if every cover of $X$ by pairwise preopen p-regular subsets has a countable refinement. 
If a bitopological space $\left(X, \tau_{1}, \tau_{2}\right)$ is pairwise almost strongly Lindelöf p-regular, then a subspace $\left(Y, \tau^{\prime}{ }_{1}, \tau^{\prime}{ }_{2}\right)$ is said to be pairwise almost strongly Lindelöf p-regular relative to $X$ if for each family $\widetilde{U}=\left\{u_{\alpha}: \alpha \in \Lambda\right\}$ consisting of pairwise preopen subsets of $X \ni Y \subseteq \bigcup_{\alpha \in \Lambda}$ precl $_{i} u_{\alpha}, \exists$ a regularlyclosed subset $F_{\alpha}$ of $X \quad \ni F_{\alpha} \subseteq u_{\alpha}$ and $Y \subseteq$ $\bigcup_{\alpha \in \Lambda} \operatorname{preint}_{i}\left(F_{\alpha}\right), \exists$ a countable subset $\left\{\alpha_{k}: k \in \mathbb{N}\right\}$ $\exists Y \subseteq \bigcup_{k \in \mathbb{N}} \operatorname{precl}_{i}\left(u_{\alpha_{k}}\right)$.

Proposition5.3: If a bitopological space $\left(X, \tau_{1}, \tau_{2}\right)$ is pairwise almost strongly Lindelöf p-regular, and $\left(Y, \tau^{\prime}{ }_{1}, \tau^{\prime}{ }_{2}\right)$ is a pairwise almost strongly Lindelöf pregular subspace of $X$, then $Y$ is pairwise almost strongly Lindelöf p-regular relative to $X$.

Proof: Let $\widetilde{U}=\left\{u_{\alpha}: \alpha \in \Lambda\right\}$ be a cover of pairwise preopen subsets of $Y, \exists$ a pairwise regularly-closed subset $F_{\alpha}$ of $X \quad \ni \quad F_{\alpha} \subseteq u_{\alpha}$ and $Y \subseteq$ $\bigcup_{\alpha \in \Lambda} \operatorname{preint}_{i}\left(F_{\alpha}\right) \forall i=1,2, \exists$ a countable subset $\left\{\alpha_{k}: k \in \mathbb{N}\right\} \ni Y \subseteq \bigcup_{k \in \mathbb{N}} \operatorname{precl}_{i}\left(u_{\alpha_{k}}\right)$.

Now, $V_{\alpha}=\operatorname{preint}_{i}\left(F_{\alpha}\right) \cap Y$ and $W_{\alpha}=u_{\alpha} \cap Y$ are pairwise preopen subsets of $Y$ and $\operatorname{precl}_{i}\left(V_{\alpha}\right)$ is a pregularly closed subset of $Y$, so $\operatorname{precl}_{i}\left(V_{\alpha}\right) \subseteq$ $\left(F_{\alpha} \cap Y\right) \subseteq u_{\alpha} \cap Y \forall \alpha \in \Lambda . \quad Y=$ $\bigcap_{\alpha \in \Lambda} \operatorname{precl}_{i} V_{\alpha}$ and $V_{\alpha} \subseteq \operatorname{int}\left(\operatorname{cl}\left(V_{\alpha}\right)\right)$ in $Y$, so $Y=$ $\mathrm{U}_{\alpha \in \Lambda} \operatorname{preint}_{i}\left(\operatorname{precl}_{j}\left(V_{\alpha}\right)\right)$, but $Y$ is a pairwise almost strongly Lindelöf p-regular subspace of $X$, so $\exists$ a countable subset $\left\{\alpha_{1}, \alpha_{2}, \ldots\right\} \quad \exists \quad Y=$ $\cup_{k \in \mathbb{N}} \operatorname{precl}_{i}\left(W_{\alpha_{k}}\right)$. Since $\operatorname{precl}_{i}\left(W_{\alpha_{k}}\right) \subseteq$ $\operatorname{precl}_{i} u_{\alpha_{k}}$, hence $Y=\bigcup_{k \in \mathbb{N}} \operatorname{precl}_{i} u_{\alpha_{k}}$. Thus, $Y$ is a pairwise almost strongly Lindelöf p-regular subspace relative to $X$.

Popsition5.4: In a bitopological space $\left(X, \tau_{1}, \tau_{2}\right)$, if every pairwise proper preclosed subset is a pairwise almost strongly Lindelöf relative to $X$, then $X$ is pairwise almost strongly Lindelöf.

Proof: Suppose that $\left\{u_{\alpha}: \alpha \in \Lambda\right\}$ be a cover of $X$ consisting of pairwise preopen subsets. Let $F_{\alpha}$ be a pairwise proper preclosed subset that is pairwise almost strongly Lindelöf relative to $X$ such that it is covered by $\left\{u_{\alpha}: \alpha \in \Lambda\right\}-F_{\alpha}$. There exists a countable subset say $\left\{\alpha_{1}, \alpha_{2}, \ldots\right\}$ such that $F_{\alpha} \subset$ $\mathrm{U}_{k \in \mathbb{N}} \operatorname{precl}_{i}\left(u_{\alpha_{k}}\right)$. Now, $X=\cup_{k \in \mathbb{N}} \operatorname{precl}_{i}\left(u_{\alpha_{k}}\right) \cup \operatorname{precl}_{i}\left(X-F_{\alpha}\right) \forall i=1,2$ which implies that $X$ is a pairwise almost strongly Lindelöf.

Proposition5.5: In a bitopological space $\left(X, \tau_{1}, \tau_{2}\right)$, if every pairwise preopen subset is a pairwise almost strongly Lindelöf relative to $X$, then the subset is pairwise almost strongly Lindelöf.
Corollary5.6: If every proper p-regularly-closed subset of a bitopological space $\left(X, \tau_{1}, \tau_{2}\right)$ is pairwise almost strongly Lindelöf p-regular, then $X$ is pairwise almost strongly Lindelöf p-regular space.

Proposition5.7: A pairwise strongly p-regular pairwise almost strongly Lindelöf is pairwise strongly Lindelöf.

A bitopological space $\left(X, \tau_{1}, \tau_{2}\right)$ is said to be a hereditarily pairwise almost strongly Lindelöf space if every subspace of $X$ is pairwise almost strongly

Lindelöf.

Proposition5.8: For hereditarily pairwise almost strongly Lindelöf spaces, the following statements are equivalent:

1. $X$ is countable discrete. 2. $X$ is hereditarily pairwise Lindelöf.

Proposition5.9:In a bitopological space $\left(X, \tau_{1}, \tau_{2}\right)$, if $U$ is a proper pairwise preopen subset of $X$ and $F$ is a proper pairwise preclosed subset of $X$ each of which is pairwise almost strongly Lindelöf relative to $X$, then $X$ is pairwise almost strongly Lindelöf. Proof: Suppose that $U$ be a pairwise preopen subset of $X$ and $\left\{u_{\alpha}: \alpha \in \Lambda\right\}$ be a cover of it consisting of pairwise preopen subsets, then $\left\{u_{\alpha}: \alpha \in \Lambda\right\} \cup(X-$ $U$ ) is a cover of $X$ consisting of pairwise preopen subsets, there exists a countable subset $\left\{\alpha_{1}, \alpha_{2}, \ldots\right\}$ such that $X=\cup_{k \in \mathbb{N}} \operatorname{precl}_{i}\left(u_{\alpha_{k}}\right) \cup \operatorname{precl}_{i}(X-U)$. Now, $U \subset \bigcup_{k \in \mathbb{N}} \operatorname{precl}_{i}\left(u_{\alpha_{k}}\right)$, hence $U$ is a pairwise almost strongly Lindelöf relative to $X$. Thus, it is a pairwise almost strongly Lindelöf subspace of $X$.

Proposition5.10: A bitopological space $\left(X, \tau_{1}, \tau_{2}\right)$ is a pairwise almost strongly Lindelöf pairwise $\mathrm{P}$-space if and only if every locally countable family of subsets of $X$ has a closure preserving property. Proof: Let that $X$ be a pairwise P-space, if $\tilde{A}=$ $\left\{A_{\alpha}: \alpha \in \Lambda\right\}$ is a locally countable family of subsets of $X$, and $\tilde{A}_{1}$ is a subfamily of $\tilde{A}$, then $\cup c l_{i}\left(A_{\alpha}\right)$ is pairwise preclosed $\forall \alpha \in \Lambda$ because $X$ is pairwise almost strongly Lindelöf pairwise P-space and $\tilde{A}$ is locally countable. Hence, $\tilde{A}$ has a closure preserving property $\forall i=1,2$.

Conversely, suppose that $\tilde{A}$ is a locally countable family, and $F_{k}$ is a pairwise preclosed subset of $X$. Let $F=\bigcup_{k \in \mathbb{N}} F_{k}$ and $x \in F_{k}$ for some $k \in \mathbb{N}$. Hence, $F_{k} \cap A_{\alpha}$ is countable since $\tilde{A}$ is locally countable. Now, $X$ has a closure preserving property, thus 


$$
\begin{aligned}
c l_{i}\left(\bigcup\left\{\left(F_{k} \cap A_{\alpha}\right): A_{\alpha} \in \tilde{A}_{1}\right\}\right) & \\
= & \bigcup \begin{array}{c}
\left\{c l_{i}\left(F_{k} \cap A_{\alpha}\right): A \in \tilde{A}_{1}\right\} \\
\forall i=1,2
\end{array}
\end{aligned}
$$

, hence $X$ is a pairwise $\mathrm{P}$-space.

6. Pairwise Weakly Strongly Lindelöf Spaces In 1996, Commoroto and Santoro [1] initiated a topological space $(X, \tau)$ that is called a weakly Lindelöf in which for each open-cover $\tilde{O}=$ $\left\{O_{\alpha}: \alpha \in \Delta\right\}$ of subsets of $X \exists$ a countable subset

$$
\left\{\alpha_{1}, \alpha_{2}, \ldots\right\} \ni X=\operatorname{cl}\left(\bigcup_{k=1}^{\infty} O_{\alpha_{k}}\right) \text {. }
$$

Definition6.1: A bitopological space $\left(X, \tau_{1}, \tau_{2}\right)$ is said to be $\tau_{i}$-weakly strongly Lindelöf space if for each pairwise preopen-cover $\tilde{O}=\left\{O_{\alpha}: \alpha \in \Delta\right\}$ of subsets of $X, \exists$ a countable subset $\left\{\alpha_{1}, \alpha_{2}, \ldots\right\} \ni X=$ $\operatorname{precl}_{i}\left(\mathrm{\cup}_{k=1}^{\infty} O_{\alpha_{k}}\right) \forall i=1,2$. If $X$ is $\tau_{1}$-weakly strongly Lindelöf and $\tau_{2}$-weakly strongly Lindelöf, then $X$ is called a pairwise weakly strongly Lindelöf bitopological space.

Proposition6.2: A bitopological space $\left(X, \tau_{1}, \tau_{2}\right)$ is pairwise weakly strongly Lindelöf if and only if for any family of pairwise preclosed subsets of $X$ say $\widetilde{F}=\left\{F_{\alpha}: \alpha \in \Delta\right\} \ni \bigcap_{\alpha \in \Delta} F_{\alpha}=\emptyset, \exists$ a countable subset $\left\{\alpha_{1}, \alpha_{2}, \ldots\right\} \ni$ preint $_{i}\left(\cap_{k=1}^{\infty} F_{\alpha_{k}}\right)=\emptyset$. Proof: Let $\widetilde{F}=\left\{F_{\alpha}: \alpha \in \Delta\right\}$ be a family of pairwise preclosed subsets of $X \ni \bigcap_{\alpha \in \Delta} F_{\alpha}=\emptyset$.

Then $X=\bigcup_{\alpha \in \Delta}\left(X-F_{\alpha}\right), \exists$ a countable subset $\left\{\alpha_{1}, \alpha_{2}, \ldots\right\} \ni$ $X=\operatorname{precl}_{i}\left(X-F_{\alpha_{k}}\right)$. So, $X-\operatorname{precl}_{i}\left(\bigcup_{k=1}^{\infty}(X-\right.$ $\left.\left.F_{\alpha_{k}}\right)\right)=\emptyset$.

That is, $\quad \operatorname{preint}_{i}\left(X-\bigcup_{k=1}^{\infty}\left(X-F_{\alpha_{k}}\right)\right)=$ preint $_{i}\left(\cap_{k=1}^{\infty} F_{\alpha_{k}}\right)=\emptyset \forall i=1,2$.

The converse is clear.

Definition 6.3: A bitopological space $\left(X, \tau_{1}, \tau_{2}\right)$ is said to be pairwise regularly weakly strongly Lindelöf space if every cover of $X$ by p-regular pairwise preopen subsets $\left\{u_{\alpha}: \alpha \in \Lambda\right\}, \exists$ a countable subset $\left\{\alpha_{1}, \alpha_{2}, \ldots\right\} \ni X=\operatorname{precl}_{i}\left(\cup_{k=1}^{\infty} u_{\alpha_{k}}\right) \forall i=$

In a bitopological space $\left(X, \tau_{1}, \tau_{2}\right)$, a subset $A$ is called pairwise weakly strongly Lindelöf relative to $X$ if for every cover $\left\{v_{\alpha}: \alpha \in \Lambda\right\}$ of $X$ consisting of pairwise preopen subsets, there exists a countable subset $\left\{\alpha_{1}, \alpha_{2}, \ldots\right\}$ such that $A \subseteq \operatorname{precl}_{i}\left(\bigcup_{k \in \mathbb{N}} v_{\alpha_{k}}\right)$.

Proposition6.4: In a bitopological space $\left(X, \tau_{1}, \tau_{2}\right)$, if $A$ is a subset of $X$, then $A$ is pairwise weakly strongly Lindelöf if and only if for every family
$\left\{F_{\alpha}: \alpha \in \mathbb{N}\right\}$ of pairwise preclosed subsets of $X$ such that $\left(\cap_{k \in \mathbb{N}} \operatorname{preint}_{i}\left(F_{\alpha_{k}}\right)\right) \cap A=\emptyset$, there exists a countable subset $\left\{\alpha_{1}, \alpha_{2}, \ldots\right\}$ such that $\left(\cap_{k \in \mathbb{N}} \operatorname{preint}_{i}\left(F_{\alpha_{k}}\right) \cap A\right)=\emptyset$.

Proposition6.5: In a bitopological space $\left(X, \tau_{1}, \tau_{2}\right)$, if $A$ is a subset of $X$ such that it is a pairwise weakly strongly Lindelöf subset relative to $X$, then $A$ is pairwise weakly strongly Lindelöf subspace of $X$. Proof: Suppose that $\left\{u_{\alpha}: \alpha \in \Lambda\right\}$ is a cover of $A$ consisting of pairwise preopen subsets of $A$, then $\left\{u_{\alpha}: \alpha \in \Lambda\right\} \cup(X-A)$ is a cover of $X$ consisting of pairwise preopen subsets, so there exists a countable subset $\quad\left\{\alpha_{1}, \alpha_{2}, \ldots\right\} \quad$ such that $A \subseteq$ preint $_{i}\left(\mathrm{U}_{k \in \mathbb{N}} u_{\alpha_{k}}\right) \forall i=1,2$. Thus, $A$ is a pairwise weakly strongly Lindelöf subspace of $X$.

\section{Conclusions}

In this article, we clarified the importance of preopen countable covers and their subfamilies and we display the role of such covers in formatting bitopological spaces we introduce and how implications are generated. Bitopological spaces we introduce are: pairwise strongly Lindelöf, pairwise nearly, pairwise almost and pairwise weakly strongly Lindelöf bitopological spaces.

\section{Acknowledgements}

The authors gratefully thank Deanship of Scientific Research and Graduate Studies in their universities and the three anonymous referees for their deductive comments and useful recommendations that helped to enhance the quality of this paper.

\section{References:}

[1] Cammaroto.F, Santoro.G, Some Counterexamples and Properties on Generalizations of Lindelöf-Spaces, International Journal of Mathematics and Mathematical Sciences, 19(4) ,1986, pp.737746.

[2] Salleh.Z, On Pairwise Nearly Lindelöf Bitopological Spaces, Far East Journal of Mathematical Sciences, 77(2), 2013, pp.147177.

[3] Hdeib.H, Sarsak.M, On Almost Lindelöf Spaces, Q \& A in General Topology, 19(1), 2001, pp.17-25.

[4] Alomary.A, Noiri.T and Noorani.M, Characterizations of strongly compact spaces, International Journal of Mathematics and Mathematical Sciences, 2009(10), 2009, pp.1-9. 
[5] Kelly.J.C, Bitopolgical Spaces. Proc. London Math. Soc. 13,1963, pp71-89.

[6] Reilly. I. L, and Vamanamurthy.M. K, On some questions concerning preopen sets, 30 , 1, 1990, pp.87-93.

[7] Hdeib.H, Pareek C.M, on Spaces in Which Lindelöf Sets Are Closed, Questions \&Answers in General Topology, 4(1), 1986, pp.67-72.

[8] Henriksen.M, Woods.R, Weak P-Space and L-closed Spaces, Questions \&Answers in General Topology, 6(2),1988, pp.201-206.

[9] Willard.S, Dissanayake.N. B, The Almost Lindelöf Degree, Canadian Mathematical Bulletin, 27(4), 1984, pp.452-455.

[10] Balasubramanian.G, On Some Generalizations of Compact Spaces, Glasnik Matematicki., 17(37), 1982, pp.367-380.

[11] Almomany.A, Chebib.M, On Almost Strongly Lindelöf and Weakly Strongly Lindelöf Spaces, Global journal of pure and applied mathematics., 15(6),2019, pp.925-934.

[12] Almomany.A, On Nearly Strongly Lindelöf Spaces, Journal of Geometry and Topology 23(2), 2019, pp.69-88.

[13] Bourbaki.N, General Topology, Part 1, Addison Wesley, Reading, Massachusetts, 1966.

[14] Frolik.Z, Generalizations of Compact and Lindelöf Spaces, Czechoslovak Mathematical Journal. 9 (84),1959, pp.172-217.

[15] Ganster.M, Preopen sets and resolvable spaces, Kyungpook Math. J. 27(2),1987, pp.135-143.

[16] Ganster.M, A note on strongly Lindelöf spaces, Soochow Journal of Mathematics, 15(1), 1989, pp.99-104.

[17] Hdeib.H and Sarsak.M, On strongly Lindelöf spaces, Q. and A. in General Topology 18(2), 2000, pp.289-298.

[18] Mashhour. A. S, Abd El-Monsef.M. $\mathrm{S}$ and El-Deeb.S. N, On precontinuous and weak precontinuous mappings, Proceedings of the Mathematical and Physical Society of Egypt 51, 1982, pp.47-53.

[19] Mashhour. A. S, Abd El-Moncef.M. E, Hasanein.I. A, and Noiri.T, Strongly compact spaces, Delta Journal of Science, 8(1), 1984, pp.30-46.

[20] Demjanovich. Yu. K, Evdokimova. T. O, Ivancova. O. N, Lebedinskii. D. M, Ponomareva. A. Y, On Wavelet Decomposition of the Singular Splines,
International Journal of Circuits, Systems and Signal Processing, 14(73), 2020, pp. 571-579.

[21] Yuri. A, Demjanovich.K, Tatjana A. Safonova, Mikhail, Terekhov.A, Belyakova.V, The Generalized Haar Spaces and Their Adaptive Decomposition, International Journal of Circuits, Systems and Signal Processing, 14(71), 2020, pp. 548-560.

\section{Contribution of individual authors to the creation of a scientific article (ghostwriting policy)}

Eman Almuhur carried out proofs of prepositions of sections 3 and 4, and she got counter examples of implications.

Manal Al-labadi carried out proofs of prepositions of sections 5 and 6.

\section{Sources of funding for research presented in a scientific article or scientific article itself \\ Funding; there is no financial support.}

\section{Creative Commons Attribution \\ License $\quad 4.0 \quad$ (Attribution $\quad 4.0$ International, CC BY 4.0)}

This article is published under the terms of the Creative Commons Attribution License 4.0 https://creativecommons.org/licenses/by/4.0/deed.en $\underline{\underline{U S}}$ 